TRANSACTIONS OF THE

AMERICAN MATHEMATICAL SOCIETY

Volume 362, Number 12, December 2010, Pages 6367-6380

S 0002-9947(2010)04887-1

Article electronically published on July 19, 2010

\title{
LEVI-FLAT HYPERSURFACES WITH REAL ANALYTIC BOUNDARY
}

\author{
JIŘÍ LEBL
}

\begin{abstract}
Let $X$ be a Stein manifold of dimension at least 3. Given a compact codimension 2 real analytic submanifold $M$ of $X$, that is the boundary of a compact Levi-flat hypersurface $H$, we study the regularity of $H$. Suppose that the CR singularities of $M$ are an $\mathcal{O}(X)$-convex set. For example, suppose $M$ has only finitely many $\mathrm{CR}$ singularities, which is a generic condition. Then $H$ must in fact be a real analytic submanifold. If $M$ is real algebraic, it follows that $H$ is real algebraic and in fact extends past $M$, even near CR singularities. To prove these results we provide two variations on a theorem of Malgrange, that a smooth submanifold contained in a real analytic subvariety of the same dimension is itself real analytic. We prove a similar theorem for submanifolds with boundary, and another one for subanalytic sets.
\end{abstract}

\section{INTRODUCTION}

Let $X$ be a Stein manifold of dimension $N$. Suppose that $M$ is a compact real submanifold of $X$ of codimension 2. We ask the following question: Does there exist a compact Levi-flat hypersurface $H$ with boundary $M$, and if so, what is the regularity of $H$ ? In this paper we address the regularity and uniqueness of $H$ in the case $M$ is real analytic. In particular, if the $\mathrm{CR}$ singularities of $M$ are an $\mathcal{O}(X)$-convex set, $H$ must in fact be a real analytic submanifold. Generically a real submanifold $M$ of codimension 2 has only finitely many $\mathrm{CR}$ singularities, which of course is an $\mathcal{O}(X)$-convex set. It is also possible to interpret these results as a regularity and uniqueness statement about a certain nonlinear partial differential equation. In [14] we saw that the real analytic case is rigid locally, and hence the present results are a natural continuation.

Malgrange [15] proved that if a smooth submanifold is contained in a real analytic subvariety of the same dimension, then the submanifold is real analytic. In $\sqrt{2}$ we prove two variations of this theorem which are of independent interest: one for subanalytic sets, and one for submanifolds with boundary.

We will suppose that $N \geq 3$. When $X$ is two dimensional, there is no CR geometric information on $M$, and the methods presented here do not apply. In particular, when $N=2$, any real analytic foliation on a real analytic submanifold of codimension 2 locally extends to a Levi-flat hypersurface. On the other hand a codimension 2 real submanifold is not in general even locally the boundary of a Levi-flat hypersurface.

Received by the editors November 13, 2007 and, in revised form, July 24, 2008.

2000 Mathematics Subject Classification. Primary 32V40, 35B65; Secondary 32W20, 32E10, $32 \mathrm{D} 15$.

(C)2010 American Mathematical Society Reverts to public domain 28 years from publication 
For a two-dimensional $X$, similar questions, both locally and globally, have been studied for many years, for example, by Bishop [6], Moser and Webster [16], Bedford 2], Huang and Krantz [11, or Bedford and Gaveau [3], among others. Our question can be interpreted as a form of a complex Plateau problem; see Bedford 2 for example. The existence question for $N \geq 3$ has been considered by Dolbeault, Tomassini and Zaitsev [10].

First we fix some terminology. In the sequel, a submanifold $M \subset X$ will always mean an embedded submanifold, i.e. the topology of $M$ is the subspace topology induced by $X$, though $M$ need not be closed in $X$. A hypersurface will mean a submanifold of codimension 1 , and a real analytic subvariety of an open set $U$ is a set closed in $U$ and locally defined by the vanishing of a family of real analytic functions. We will say that $V \subset \mathbb{R}^{N}$ is a local real analytic subvariety if there exists an open set $U$ such that $V$ is a real analytic subvariety of $U$. A submanifold will be called real algebraic if it is contained in a real algebraic subvariety (a subvariety defined by the vanishing of real polynomials) of the same dimension.

We give the following definitions assuming $X=\mathbb{C}^{N}$ for clarity. The definitions are local in character and carry over to an arbitrary Stein manifold in a straightforward way.

Let $M \subset \mathbb{C}^{N}$ be a real submanifold. Let $J$ be the complex structure on $\mathbb{C}^{N}$, and let $T_{p}^{c} M=J\left(T_{p} M\right) \cap T_{p} M . \quad M$ is said to be $C R$ near $p$ if there exists a neighbourhood $U$ of $p$ such that $\operatorname{dim} T_{q}^{c} M$ is constant as $q$ varies in $U$. The points near which $M$ is CR will be denoted by $M_{C R}$, and the complement of $M_{C R}$ we will call the $\mathrm{CR}$ singular points. The set of $\mathrm{CR}$ singular points is a real analytic subvariety of $M$.

A set $H \subset \mathbb{R}^{n}$ is a $C^{k}$ hypersurface with boundary if there is a subset $\partial H \subset H$, such that $\partial H \subset H, H \backslash \partial H$ is a $C^{k}$ hypersurface (submanifold of codimension 1 ), and for each point $p \in \partial H$, there exists a neighbourhood $p \in U \subset \mathbb{R}^{n}$, a $C^{k}$ diffeomorphism $\varphi: U \rightarrow \mathbb{R}^{n}$, such that $\varphi(H \cap U)=\left\{x \in \mathbb{R}^{n} \mid x_{n-1} \geq 0, x_{n}=0\right\}$, and such that $\varphi(\partial H \cap U)=\left\{x \in \mathbb{R}^{n} \mid x_{n-1}=0, x_{n}=0\right\}$. Hence, $\partial H$ is a $C^{k}$ submanifold of codimension 2 in $\mathbb{R}^{n}$. We will call $H^{o}:=H \backslash \partial H$ the interior of $H$. A $C^{k}(k \geq 2)$ hypersurface $H \subset \mathbb{C}^{N} \cong \mathbb{R}^{2 N}$ is said to be Levi-flat if the bundle $T^{c} H$ is involutive. If $H$ is a hypersurface with boundary, then we will say it is Levi-flat when $H^{\circ}$ is Levi-flat.

Our first result is the following. For a Stein manifold $X$, let $\mathcal{O}(X)$ denote the holomorphic functions on $X$. For a compact set $S$, let $\hat{S}$ denote the $\mathcal{O}(X)$-convex hull of $S$.

Theorem 1.1. Let $X$ be a Stein manifold of dimension $N \geq 3$. Let $M \subset X$ be a compact real analytic submanifold of codimension 2. Suppose there exists a compact connected $C^{\infty}$ Levi-flat hypersurface $H$ with boundary, such that $\partial H=M$.

Let $S$ be the set of $C R$ singularities of $M$. If $S=\hat{S}$, then $H^{\circ}=H \backslash M$ is real analytic. Further, $H$ is the unique compact connected Levi-flat $C^{\infty}$ hypersurface with boundary $M$.

In particular if $S$ is a finite set, then $S=\hat{S}$ and $H^{o}$ is real analytic and unique. Since $M$ is an oriented codimension 2 compact submanifold, it is a standard result that the condition of only isolated CR singularities (hence finitely many) is the generic situation. We need only look at the Gauss map of the manifold and apply Thom's transversality theorem. See Lai [12] for example. 
We will see that $H$ actually extends as a real analytic submanifold past all the $\mathrm{CR}$ points, though at $\mathrm{CR}$ singularities the picture is not clear.

Let us interpret this result as a regularity and uniqueness statement for a certain partial differential equation. Suppose that $\rho$ is a function with $d \rho \neq 0$. The hypersurface $\{\rho=0\}$ is Levi-flat if and only if the Levi form vanishes [1,7,8. That is equivalent to the complex Hessian vanishing on all holomorphic vectors tangent to the hypersurface. Hence $\{\rho=0\}$ is Levi-flat if and only if the complex bordered Hessian is of rank two on the hypersurface. In other words, $\{\rho=0\}$ is Levi-flat if and only if

$$
\operatorname{rank}\left[\begin{array}{cc}
\rho & \rho_{z} \\
\rho_{\bar{z}} & \rho_{z \bar{z}}
\end{array}\right]=2 \quad \text { for all points on }\{\rho=0\} .
$$

As (1) implies the determinant of the complex bordered Hessian is zero, the equation is related to equations of the complex Monge-Ampère type.

We can also think of the hypersurface as a graph of a certain function. This leads us to the following somewhat more complicated differential equation. Let $\Omega \subset \mathbb{C}^{N-1} \times \mathbb{R}$ be a domain and let us call the coordinates $(z, s) \in \mathbb{C}^{N-1} \times \mathbb{R}$, and suppose $u: \Omega \rightarrow \mathbb{R}$ is a function that satisfies

$$
\begin{aligned}
& u_{z_{j}}\left(-i+u_{s}\right) u_{s \bar{z}_{j}}+u_{\bar{z}_{j}}\left(i+u_{s}\right) u_{s z} \\
& \quad+u_{z_{j}} u_{\bar{z}_{j}} u_{s s}-\left(1+u_{s}^{2}\right) u_{z_{j} \bar{z}_{j}}=0
\end{aligned}
$$

We can consider the graph of this function to lie in $\mathbb{C}^{N}$ by looking at the set defined by $\operatorname{Im} w=u(z, \bar{z}, \operatorname{Re} w)$. The graph is then a Levi-flat hypersurface, as $\rho(z, \bar{z}, w, \bar{w})=\operatorname{Im} w-u(z, \bar{z}, \operatorname{Re} w)$ satisfies (11).

Now consider the following boundary value problem. Suppose that $\Omega$ has real analytic boundary. Let $g: \partial \Omega \rightarrow \mathbb{R}$ be a real analytic function, and suppose there exists a solution $u \in C^{\infty}(\bar{\Omega})$ that satisfies (2) and $g=\left.u\right|_{\partial \Omega}$. Further, impose the generic condition that at most at finitely many points is $\partial \Omega$ tangent to a line $\left\{s=s_{0}\right\}$, for some $s_{0} \in \mathbb{R}$. Then Theorem 1.1 tells us that $u$ is real analytic on $\Omega$. Further, $u$ is the unique solution in $C^{\infty}(\bar{\Omega})$.

Let us now look at Theorem 1.1 when $M$ is a real algebraic submanifold. In this case, we will be able to extend the hypersurface $H$ past $M$ even near CR singularities; see Lemma 4.1. Using this lemma we extend Theorem 1.1 as follows.

Theorem 1.2. Let $M \subset \mathbb{C}^{N}, N \geq 3$, be a compact real algebraic submanifold of codimension 2. Suppose there exists a compact connected $C^{\infty}$ Levi-flat hypersurface $H$ with boundary, such that $\partial H=M$.

If the set of $C R$ singularities of $M$ is polynomially convex, then there exists a real algebraic Levi-flat hypersurface $\mathcal{H}$ (without boundary) such that $H \subset \mathcal{H}$.

Theorems 1.1 and 1.2 do not hold as stated when $N=2$. For the uniqueness, see Example 6.2. For more discussion and examples on the regularity in two dimensions, see the Appendix in [2].

More background details about CR geometry and some of the methods employed here can be found in the books [1,7,8].

In $\$ 2$ we discuss two variations on a theorem of Malgrange that will be needed in the proofs. In $₫ 3$ we prove Theorem 1.1. In $\$ 4$ we discuss the real algebraic case 
and prove Theorem 1.2 In $\$ 5$ we consider hypersurfaces with isolated singularities. In $\sqrt[6]{6}$ we look at some examples.

The author would like to acknowledge Peter Ebenfelt for many useful discussions while preparing these results. Also, the author would like to acknowledge John D'Angelo, Edward Bierstone, and Dmitri Zaitsev for their useful comments on the results and the manuscript. The author would like to acknowledge Alexander Tumanov for suggesting to consider singular hypersurfaces as is done in \$5.

\section{Two VARiations on the TheOREM of Malgrange}

Malgrange proved that a smooth submanifold contained in a real analytic subvariety of the same dimension is itself real analytic. See 1, Theorem 5.5.32 or 15, Chapter VI, Proposition 3.11. We will prove two variations on this theorem, one for subanalytic sets, and one for submanifolds with boundary. As these results are of independent interest, we will prove these theorems for arbitrary codimension even though we will only apply them in the hypersurface case. The first theorem we wish to prove is a subanalytic version of Malgrange's theorem. The class of semianalytic sets is the smallest class containing sets of the form $\{f>0\}$ for real analytic functions $f$ that is closed under finite union, intersection and complement. A set is subanalytic if it is locally a projection of a relatively compact semianalytic set. See Bierstone and Milman [4] for more information about subanalytic and semianalytic sets.

Theorem 2.1. Suppose that $Y \subset \mathbb{R}^{N}$ is a $C^{\infty}$ submanifold, and $S \subset \mathbb{R}^{N}$ a subanalytic set of the same dimension as $Y$, such that $Y \subset S$. Then $Y$ is real analytic.

Next we want to prove a version for submanifolds with boundary.

Theorem 2.2. Suppose that $Y \subset \mathbb{R}^{N}$ is a $C^{\infty}$ submanifold with boundary, and $V \subset \mathbb{R}^{N}$ a local real analytic subvariety of the same dimension as $Y$, such that $Y \subset V$. Then there exists a real analytic submanifold $\mathcal{Y}$ of the same dimension as $Y$ such that $Y \subset \mathcal{Y}$.

In particular, such a $Y$ extends uniquely past its boundary as a real analytic submanifold. Combining the ideas of the proofs we can see that we could replace "local real analytic subvariety" in Theorem 2.2 with "subanalytic set."

As we will be concerned with convergence we will require the following classical theorem. A proof can be found for example in [1] (Theorem 5.5.30).

Theorem 2.3. Suppose $T(x)$ is a formal power series in $x \in \mathbb{R}^{N}$. Suppose $T(t v)$ is a convergent power series in $t \in \mathbb{R}$ for all $v \in \mathbb{R}^{N}$. Then $T$ is convergent.

In particular, one corollary of this theorem is that if $f(x)$ is a $C^{\infty}$ function, and if there exists a neighborhood $0 \in U \subset \mathbb{R}^{N}$ such that $t \mapsto f(t x)$ is real analytic whenever $t x \in U$, then $f$ is real analytic.

Proof of Theorem 2.1. Let $Y$ and $S$ be as in the statement of Theorem 2.1. First suppose that $Y$ is a hypersurface. Without loss of generality, let $(x, y) \in \mathbb{R}^{m} \times \mathbb{R}$ be our coordinates and suppose that $Y$ is defined by $y=f(x)$ for a $C^{\infty}$ function $f$, with $f(0)=0$. We need only to prove that $Y$ is real analytic near 0 .

We restrict to a line and look at the graph of the function $t \mapsto f(t x)$. The graph is an intersection of $Y$ and a 2-dimensional plane $P_{x}$. Suppose that $S \cap P_{x}$ was of dimension 2. Let $\tilde{S}=\overline{S \backslash P_{x}}$, which is again a subanalytic set containing $Y$ 
and of the same dimension as $Y$. A subanalytic set is a locally finite union of real analytic submanifolds. Further, there is a sequence $\left\{x_{j}\right\}$ converging to $x$ such that $\operatorname{dim}\left(S \cap P_{x_{j}}\right)=1$. Hence, $\tilde{S} \cap P_{x}$ must be of dimension 1 .

Hence, the graph of $t \mapsto f(t x)$ is contained in a subanalytic set of dimension 1. By a theorem of Łojasiewicz (see [4, Theorem 6.1), a one-dimensional subanalytic set is semianalytic and hence contained in a real analytic subvariety of the same dimension. We can now apply the standard version of the theorem of Malgrange to see that $t \mapsto f(t x)$ must be real analytic. By the discussion after Theorem 2.3, we know that $f$, and therefore $Y$ itself, must be real analytic near 0 .

Now suppose that $Y$ is of higher codimension. That is, let $(x, y) \in \mathbb{R}^{m} \times \mathbb{R}^{n}$ be our coordinates and suppose that $Y$ is defined by $y=f(x)$ for a $C^{\infty}$ mapping $f$, with $f(0)=0$. Write $f=\left(f_{1}, \ldots, f_{n}\right)$. Pick $1 \leq j \leq n$, and let $\tilde{Y}$ be the submanifold defined by $y_{j}=f_{j}(x)$ in $\mathbb{R}^{m} \times \mathbb{R}$. Take $\pi$ to be the projection $(x, y) \mapsto$ $\left(x, y_{j}\right)$. As $S$ has dimension $m, \pi(S)$ is a subanalytic set of dimension at most $m$. Because $\pi(S)$ contains $\tilde{Y}$, it must be of dimension $m$ exactly. Applying the result for hypersurfaces, we see that $f_{j}$ is real analytic near 0 for all $j$. Therefore, $Y$ is real analytic near 0 .

To prove Theorem 2.2, it is enough to show that near every point $p \in Y, Y$ is contained in a real analytic submanifold of the same dimension. The techniques used in this proof will be similar to those in [1. If $p$ is not on the boundary, the statement follows from the standard statement of the theorem of Malgrange. What we will prove, therefore, is the case when $p \in \partial Y$. We can assume that near $p$, the boundary of $Y$ is real analytic, since we can always find a smaller submanifold $\tilde{Y} \subset Y$ of equal dimension and with real analytic boundary such that $p \in \tilde{Y}$. To find $\tilde{Y}$ we intersect $Y$ and $V$ with a real analytic nonsingular hypersurface $B$ transversal to $Y$ such that $B \cap Y$ is a smooth submanifold through $p$ and is contained in $B \cap V$, which is of the same dimension as $B \cap Y$. Take $B \cap Y$ to be the new boundary, which is real analytic by the standard statement of the theorem. If $\tilde{Y}$ is real analytic at $p$, then it uniquely extends as a real analytic submanifold past $p$. $Y$ must therefore be contained in this extension by analytic continuation, since we know that $Y$ is real analytic in the interior.

We will assume that $p$ is the origin. We first prove the codimension 1 case. Assume that $Y$ is a hypersurface with boundary. After straightening the boundary of $Y$, applying the Weierstrass preparation theorem to $V$ at 0 , and rescaling, we see that it suffices to prove the following lemma. We will denote by $\mathbb{B}_{N} \subset \mathbb{R}^{N}$ the unit ball centered at 0 .

Lemma 2.4. Put $\Omega=\mathbb{B}_{N} \cap\left\{x \in \mathbb{R}^{N} \mid x_{N} \geq 0\right\}$. Let

$$
P(x, y)=y^{k}+c_{k-1}(x) y^{k-1}+\cdots+c_{0}(x),
$$

where $c_{j}$ are real analytic functions on $\mathbb{B}_{N}$, and $c_{j}(0)=0$ for $j=0, \ldots, k-1$. Suppose $f: \Omega \rightarrow \mathbb{R}$ is a $C^{\infty}$ function such that

$$
f(0)=0 \text {, and } P(x, f(x))=0 \text { for } x \in \Omega .
$$

Then $f$ is real analytic near 0 .

What must be shown is that the formal Taylor series of $f$ converges for $x$ near 0 , and converges to $f$ whenever such $x$ is in $\Omega$. We will want to apply Theorem 2.3. and so we will want to reduce the proof of Lemma 2.4 to the one-dimensional case. 
Here we will use the following statement of the Puiseux theorem. A proof can be found for example in [5] or [1].

Theorem 2.5 (Puiseux). Let $P(x, y)=y^{k}+c_{k-1}(x) y^{k-1}+\cdots+c_{0}(x)$, where $c_{j} \in \mathbb{C}\{x\}$ and $c_{j}(0)=0$ for $j=0, \ldots, k-1$. Suppose that $P$ is irreducible. Then there exists $a \in \mathbb{C}\{x\}$ such that

$$
P\left(t^{k}, y\right)=\prod_{j=1}^{k}\left(y-a\left(\omega^{j} t\right)\right),
$$

where $\omega=e^{i 2 \pi / k}$.

In one dimension, Lemma 2.4 reduces to the following statement.

Lemma 2.6. Let $P(x, y)=y^{k}+c_{k-1}(x) y^{k-1}+\cdots+c_{0}(x)$, where the $c_{j}$ are real analytic functions on $(-1,1)$ such that $c_{j}(0)=0$. Suppose $f:[0,1) \rightarrow \mathbb{R}$ is a $C^{\infty}$ function such that $f(0)=0$ and $P(x, f(x))=0$ for $x \in[0,1)$. Then $f$ is real analytic.

Proof. By the theorem of Malgrange, we know that $f$ is real analytic on $(0,1)$. Hence the graph of $f$ must lie in a single branch of the zero locus of $P$, and we can assume that $P$ is irreducible. If we plug in $f\left(t^{k}\right)$ for $y$ into (5), we see that there exists a $j$ such that $f\left(t^{k}\right)=a\left(\omega^{j} t\right)$, for $t \geq 0$. Hence the Taylor series of $t \mapsto f\left(t^{k}\right)$ at 0 is equal to the power series expansion of $t \mapsto a\left(\omega^{j} t\right)$. Therefore the Taylor series of $f$ also converges, and converges to $f$.

Proof of Lemma 2.4, Let $T_{f}$ be the formal Taylor series of $f$ at the origin. Pick an arbitrary $v \in \mathbb{R}^{N}$, and without loss of generality suppose that $v_{N} \geq 0$ and $\|v\|=1$. The function $g(t)=f(t v)$ is a $C^{\infty}$ function for $t \in[0, \epsilon)$ for some $\epsilon>0$. By Lemma 2.6. the Taylor series of $g$ at 0 converges, and applying Theorem 2.3 we see that $T_{f}$ converges as well. Suppose it converges for $\|x\|<\delta$. The $\delta$ therefore no longer depends on $x$. Lemma 2.6 tells us that the Taylor series of $g$ converges to $f(t v)$ for $t \in[0, \delta)$, and as it converges for all $v$ as above, $T_{f}$ converges to $f$ for $\|x\|<\delta$ and $x_{N} \geq 0$, as desired.

To finish the proof of Theorem 2.2. we only need to reduce to the hypersurface case. Let $Y$ and $V$ be as in the statement of the theorem. After a linear change of coordinates we only need to apply the partial generalization of the Weierstrass Preparation Theorem to $V$ (see for example [1, Theorem 5.3.9). That is, we note that locally near 0 there exists a subvariety $\tilde{V}$ of the same dimension as $V$, such that $V \cap U \subset \tilde{V} \cap U$ for some small neighbourhood $U$ of 0 , and such that $\tilde{V}$ is defined in the coordinates $(x, y) \in \mathbb{R}^{m} \times \mathbb{R}^{n}$ by

$$
y_{k}^{d_{k}}+\sum_{j=0}^{d_{k}-1} a_{j k}(x) y_{k}^{j} \quad \text { for } k=n, \ldots, N
$$

where $a_{j k}$ are real analytic functions vanishing at 0 . Therefore, as we can assume that $Y$ is a graph over the $x$ coordinates, we see that the theorem follows by applying Lemma 2.4 


\section{Real anAlytic Regularity}

The following more general statement implies the regularity part of Theorem 1.1. Let $\mathcal{O}(X)$ denote the holomorphic functions on $X$.

Theorem 3.1. Let $M \subset X$ be a compact real analytic submanifold of codimension 2. Suppose there exists a compact connected $C^{\infty}$ Levi-flat hypersurface $H$ with boundary such that $\partial H=M$.

Let $S$ be the set of $C R$ singular points of $M$, and $\hat{S}$ the $\mathcal{O}(X)$-convex hull of $S$. Then $H \backslash(M \cup \hat{S})$ is a real analytic submanifold.

If a hypersurface $H$ is Levi-flat, then it is locally foliated by complex hypersurfaces. This foliation is called the Levi foliation. The smallest germ (in terms of dimension) of a CR submanifold $W$ of $M$ through $p$ such that the $T_{q}^{c} W=T_{q}^{c} M$ is called the local $C R$ orbit at $p$, and is guaranteed to exist by a theorem of Nagano in case $M$ is real analytic, and by a theorem of Sussmann in case $M$ is smooth. The following two results have been previously known in some form. Proofs using similar notation as the present paper can be found in [14.

Lemma 3.2. Suppose $H \subset \mathbb{C}^{N}, N \geq 2$, is a $C^{2}$ Levi-flat hypersurface with boundary, $M \subset \mathbb{C}^{N}$ is a $C^{\infty}$ submanifold of codimension 2 , and $M=\partial H$. If $M$ is $C R$ near $p \in M$, then the local $C R$ orbit of $M$ at $p$ is of positive codimension in $M$ (i.e. $M_{C R}$ is nowhere minimal).

Lemma 3.3. Suppose $H \subset \mathbb{C}^{N}, N \geq 3$, is a $C^{2}$ Levi-flat hypersurface with boundary, $M \subset \mathbb{C}^{N}$ is a real analytic submanifold of codimension 2 , and $M=\partial H$. Suppose that near $p \in M_{C R}$, the local $C R$ orbits are all of codimension 1 in $M$. Then there exists a neighbourhood $U$ of $p$ such that $(U \cap H) \subset \mathcal{H}$, where $\mathcal{H}$ is the unique Levi-flat real analytic hypersurface in $U$ that contains $M \cap U$.

At a key point in the proof we will require the following theorem by Rossi (see Theorem 4.7 in [17]). Let $K$ be a compact subset of $X, \mathcal{O}(K)$ the algebra of functions holomorphic on a neighbourhood of $K$, and $A(K)$ the closure of $\mathcal{O}(K)$ in the uniform norm on $K$. Let $M(\mathcal{A})$ be the set of peak points of the algebra $\mathcal{A}$, and $\Gamma(\mathcal{A})$ be the Silov boundary of the algebra $\mathcal{A}$. In particular, $p \in M(\mathcal{O}(K))$ if there exists an $f \in \mathcal{O}(K)$ such that $f(p)=1$ and $|f(q)|<1$ for $q \neq p$.

Theorem 3.4 (Rossi). Suppose $K \subset X$ is compact and $K=\bigcap_{1}^{\infty} U_{k}$, where the $U_{k}$ are Stein submanifolds of $X$. Then $M(\mathcal{O}(K))$ is dense in $\Gamma(A(K))$.

In the same paper (Lemma 2.9) Rossi also shows that if $K$ is convex with respect to the holomorphic functions on $X$, then $K$ satisfies the hypothesis in Theorem 3.4. i.e. $K=\bigcap_{1}^{\infty} U_{k}$ for Stein submanifolds $U_{k}$ of $X$.

Proof of Theorem 3.1. Let $X$ be a Stein manifold of dimension $N \geq 3$. Assume that $M$ and $H$ are as in the statement of the theorem. That is, $M \subset X$ is a compact real analytic submanifold of codimension 2 and $H$ is a connected compact $C^{\infty}$ Levi-flat hypersurface with boundary such that $\partial H=M$.

A priori, the local CR orbit of $M_{C R}$ at any particular point can be either codimension 0,1 , or 2 in $M$. We see from Lemma 3.2 that the local CR orbit cannot be of codimension 0 in $M$. If some local CR orbit is of codimension 2 in $M$, then it is in fact a germ of a complex submanifold. By the theorem of Diederich and Fornaess [9, no compact real analytic submanifold contains a germ of a nontrivial complex 
submanifold. If $N \geq 3$, then such a local $\mathrm{CR}$ orbit would in fact be nontrivial. Hence if $M$ is a compact real analytic boundary of a Levi-flat hypersurface, then all the local $\mathrm{CR}$ orbits at all points of $M_{C R}$ must be of codimension 1 in $M$.

We will call $B \subset H$ the set of points where $H$ is not real analytic. First, it is obvious that $B$ is a compact set. By applying Lemma 3.3 , we can see that $H$ is real analytic, and thus extends past $M$, near all the $\mathrm{CR}$ points of $M$. Hence $B$ must be a proper closed subset of $H$ such that $B \cap M_{C R}=\emptyset$. Recall that $S$ is the set of CR singular points of $M$ and $\hat{S}$ is the $\mathcal{O}(X)$-convex hull of $S$.

Lemma 3.5. Let $B$ and $S$ be compact subsets of $X$. If $B \backslash \hat{S}$ is nonempty, then there exists a point $p \in B \backslash \hat{S}$, a neighbourhood $U \subset X$ of $p$, and a holomorphic function $f$ defined on $U$, such that

$$
f(p)=1 \text { and }|f(z)|<1 \text { for all } z \in B \cap U \backslash\{p\} .
$$

Proof. Let us first take $\hat{B}$ to be the convex hull with respect to the holomorphic functions on $X$. We will prove a stronger statement than we need by showing there must exist a point $p \in M(\mathcal{O}(\hat{B})) \cap(B \backslash \hat{S})$.

The Šilov boundary $\Gamma(A(\hat{B}))$ is a subset of $B$. Further, the $\mathcal{O}(X)$-convex hull of $\Gamma(A(\hat{B}))$ is $\hat{B}$. We assume that $B \backslash \hat{S}$ is nonempty; therefore $\hat{B} \backslash \hat{S}$ is nonempty. Hence, there exists a $q \in \Gamma(A(\hat{B}))$ such that $q \in B \backslash \hat{S}$.

We apply Theorem 3.4 to $\hat{B}$ to find that $M(\mathcal{O}(\hat{B}))$ is dense in $\Gamma(A(\hat{B}))$. Since $\hat{S}$ is closed and $q \notin \hat{S}$, there exists a point $p \in M(\mathcal{O}(\hat{B}))$ near $q$, such that $p \notin \hat{S}$. Since $M(\mathcal{O}(\hat{B})) \subset \Gamma(A(\hat{B})) \subset B$, we see that $p \in M(\mathcal{O}(\hat{B})) \cap(B \backslash \hat{S})$.

Assume for a contradiction that $B \backslash \hat{S}$ was nonempty, and that $p$ exists by Lemma 3.5. Do note that $p \in H^{o}$ because $B \backslash S \subset H^{o}$. Suppose that $U$ is small enough that all leaves of the Levi foliation of $H \cap U$ are closed. If $L \subset H \cap U$ is the closed leaf of the Levi foliation of $H \cap U$ such that $p \in L$, then the set $\{|f| \geq 1\} \cap L$ has $p$ as a limit point by the maximum principle. Hence $L \backslash B$ also has a limit point $p$.

Claim 3.6. For a perhaps smaller neighbourhood $U$ of $p$, there exist open sets $V \subset W \subset \mathbb{C}^{N-1} \times \mathbb{R}$, with $0 \in W$, and a mapping $\varphi: W \rightarrow X$ that is holomorphic in the first $N-1$ variables, such that

(i) $\varphi(0)=p$,

(ii) $\varphi$ is one-to-one,

(iii) $\left.\varphi\right|_{V}$ is real analytic and a diffeomorphism onto its image,

(iv) if $L$ is the leaf of the Levi foliation of $H \cap U$ through $p$, then $\varphi(V)$ contains one of the connected components of $L \backslash B$,

(v) $p \in \overline{\varphi(V)}$.

Proof. Assume that $p=0$. Further, we can suppose that $U$ is small enough such that we can make a local change of coordinates to assume that the leaf $L$ of the Levi foliation through 0 is defined by $\left\{z_{1}=0\right\} \cap U$. We can assume that $U$ is perhaps even smaller, such that all the leaves of the Levi foliation of $H \cap U$ are connected graphs over $L$. For any leaf $L^{\prime}$ of the foliation we have a well-defined holomorphic function $\zeta \mapsto \psi_{L^{\prime}}(\zeta)$, where $\zeta \in \mathbb{C}^{N-1}$, whose graph over $L$ is $L^{\prime}$.

Pick a point $q$ on some connected component of $L \backslash B$, whose closure includes p. Such a $q$ exists for example on the set $\{|f|=1\} \cap L$. As $H$ is real analytic near $q, H$ is locally defined by $\left\{\operatorname{Im} w_{1}=0\right\}$ for some local coordinates $w$. We can now define $\varphi(\zeta, t):=\left(\zeta, \psi_{L_{t}}(\zeta)\right)$, where $L_{t}$ is the leaf that near $q$ is locally defined by 
$\left\{w_{1}=t\right\}$. What is left to do is to find $V$ and show that $\left.\varphi\right|_{V}$ is a diffeomorphism onto the right set.

Obviously, $\varphi$ is real analytic near $\varphi^{-1}(q)$. We can cover the connected component of $L \backslash B$ that contains $q$ by a locally finite collection of neighbourhoods $V_{j} \subset L$, such that for every point in $V_{j}$ a local change of coordinates as above is possible. As we can identify $L$ with an open set in $\mathbb{C}^{N-1}$, we will identify $V_{j} \subset L \subset \mathbb{C}^{N}$ and the corresponding set in $\mathbb{C}^{N-1}$. It is not hard to see that if $V_{j}$ and $V_{k}$ overlap and $\varphi$ is analytic on $V_{j} \times(-\epsilon, \epsilon)$, then perhaps for some smaller $\epsilon^{\prime}, \varphi$ is real analytic on $\left(V_{j} \cup V_{k}\right) \times\left(-\epsilon^{\prime}, \epsilon^{\prime}\right)$. It is also not hard to see that if the derivative in the last variable does not vanish on $V_{j} \times\{0\}$, then it does not vanish on $V_{k} \times\{0\}$.

Let $V=\bigcup_{j} V_{j} \times\left(-\epsilon_{j}, \epsilon_{j}\right)$. $\varphi$ is obviously one-to-one and with a nonvanishing Jacobian on $V$, hence is a diffeomorphism onto its image.

As $\left.\varphi\right|_{V}$ is real analytic, we can complexify the last variable in $\left.\varphi\right|_{V}$. We obtain a holomorphic mapping $\tilde{\varphi}$ defined on an open set $\tilde{V} \subset \mathbb{C}^{N-1} \times \mathbb{C}$, where we can think of $V \subset \tilde{V}$, such that $\left.\varphi\right|_{V}=\left.\tilde{\varphi}\right|_{V}$.

Note that $0 \in \partial \tilde{V}$. We will show that $\tilde{V}$ is not Hartogs pseudoconvex at 0 . Let $L$ be the leaf of the Levi foliation of $H \cap U$ at $p$. We can assume $U$ is small enough such that $L$ is closed in $H \cap U$. Take the set $\{|f|=1\} \cap L$; this is a Levi-flat real analytic subvariety of $L$ and intersects $B$ only at $p$. For all $\theta$ sufficiently close to 0 , the set $\left\{f=e^{i \theta}\right\} \cap L$ is a nontrivial proper complex subvariety of $L$ and intersects $B$ only when $\theta=0$ and then it intersects $B$ only at $p$. Therefore we can find a sequence of closed analytic discs $\Delta_{j} \subset L \backslash B$ such that $\overline{\bigcup_{j} \partial \Delta_{j}} \subset L \backslash B$, but $\overline{\bigcup_{j} \Delta_{j}} \cap B=\{p\}$. Hence $L \backslash B$ is not psuedoconvex at 0 and hence $\tilde{V}$ cannot be pseudoconvex at 0 .

Since $\tilde{V}$ is not Hartogs pseudoconvex at 0 , then $\tilde{\varphi}$ extends to a holomorphic mapping $\Phi$ defined on a larger set, in particular in a neighbourhood of 0 . By uniqueness of analytic continuation along the leaves of the Levi foliation, we see that $\Phi$ agrees with $\varphi$ on $W$ near 0 , and hence $\varphi$ is real analytic near 0 .

Thus near $p, H$ is a subanalytic set. It is also a $C^{\infty}$ hypersurface, and by Theorem 2.1. $H$ is real analytic at $p$. Hence $p \notin B$, but that would be a contradiction. $B \backslash \hat{S}$ must have been empty to begin with, and we are done.

The technique in the proof of Theorem 3.1 suggests other similar results.

Proposition 3.7. Suppose $H \subset X$ is a $C^{\infty}$ Levi-flat hypersurface (without boundary). Suppose $K \subset H$ is a compact set such that $H \backslash K$ is a real analytic submanifold. Then $H$ is a real analytic submanifold.

To prove the proposition, we again let $B \subset K$ be the set where $H$ is not real analytic. Let $S=\emptyset$ and apply Lemma 3.5 to find a $p \in B$, a neighbourhood $U$ and a peaking function $f$. Then we follow the proof of Theorem 3.1 from that point on to show that $p \notin B$, thereby showing that $B$ must have been empty to begin with.

Proof of the uniqueness in Theorem 1.1. Let $M$ be as in the statement of the theorem. Again let $S$ be the set of CR singular points of $M$. Suppose $H$ and $H^{\prime}$ are two compact connected Levi-flat $C^{\infty}$ hypersurfaces with boundary $M$. As we assume $S=\hat{S}$, Theorem 3.1 shows that $H^{o}$ and $H^{\prime o}$ are both real analytic. By Lemma 3.3 , for a point $q \in M_{C R}$, there is a neighbourhood $U_{q} \subset X$ of $q$ and a unique Levi-flat hypersurface $\mathcal{H}$ that contains both $H \cap U_{q}$ and $H^{\prime} \cap U_{q}$. We can assume that both 
$H \cap U_{q}$ and $H^{\prime} \cap U_{q}$ are connected. There are two possibilities:

$$
H \cap U_{q}=H^{\prime} \cap U_{q} \text { or } H \cap H^{\prime} \cap U_{q}=M \cap U_{q} .
$$

In the first case, because $H$ and $H^{\prime}$ are path connected and real analytic, unique continuation implies that $H=H^{\prime}$. Let us therefore suppose that $H \cap H^{\prime} \cap U_{q}=$ $M \cap U_{q}$ for all points $q \in M_{C R}$.

The compact set $H \cup H^{\prime}$ is therefore such that $\left(H \cup H^{\prime}\right) \backslash S$ is a Levi-flat subvariety of codimension 1 of $X \backslash S$. Now note that $\hat{S}=S$. If we apply Lemma 3.5, there exists a point $p \in H \cup H^{\prime}$ such that $p \notin S$, and a peaking function $f$. That is, there exists a neighbourhood $V$ of $p$, and a function $f$ holomorphic in $V$ such that $|f(z)|<1$ for $z \in V \cap\left(H \cup H^{\prime}\right) \backslash\{p\}$ and $f(p)=1$. Simply take $B$ to be $H \cup H^{\prime}$ in Lemma 3.5. Since $\left(H \cup H^{\prime}\right) \backslash S$ is locally a union of complex hypersurfaces, the existence of $p$ and $f$ violates the maximum principle.

\section{Real algebraic eXtension}

Ideally, one would want to have an extension result in the same spirit as those in 14, but for CR singular points. The following lemma is a small step in this direction and will be sufficient to prove Theorem [1.2, As the following lemma is local, note that $H$ need not be closed. Hence, $\partial H$ need not include all the points in $\bar{H}$ even though $H$ is embedded.

Lemma 4.1. Let $0 \in M \subset \mathbb{C}^{N}, N \geq 3$, be a real-algebraic submanifold of codimension 2, such that 0 is a CR singular point of $M$ and not all local CR orbits of $M_{C R}$ are codimension 2 in $M$. Suppose there exists a connected $C^{\infty}$ hypersurface $H$ with boundary, such that $H^{\circ}$ is real analytic, $H$ is Levi-flat and $\partial H=M$.

Then there exists a local algebraic change of coordinates near 0 such that $M$ is locally given in $(z, w) \in \mathbb{C}^{N-1} \times \mathbb{C}$ by an equation of the form

$$
w=\varphi(z, \bar{z}),
$$

where $\varphi$ is real valued.

Hence, $H$ is locally given by the equation $\operatorname{Im} w=0$ and therefore extends past $M$ near 0 . For the proof, we will require the following theorem. Nowhere minimal means that all local $\mathrm{CR}$ orbits of $M$ are of positive codimension in $M$. We say that $M$ is generic if and only if $T_{p} M+J\left(T_{p} M\right)=T_{p} \mathbb{C}^{N}$ for all $p \in M$, where $J$ is the complex structure on $\mathbb{C}^{N}$. By $H^{*}$, we mean the nonsingular points of $H$ of top dimension (i.e. codimension 1 in $\mathbb{C}^{N}$ ).

Theorem 4.2 (see 13). Let $(M, 0) \subset \mathbb{C}^{N}$ be a germ of a real algebraic nowhere minimal generic submanifold of codimension 2. Then there exists an irreducible germ of a Levi-flat real algebraic subvariety $(H, 0)$ of codimension 1 , such that for some representatives $M$ and $H$ of the germs we have that $M \subset \overline{H^{*}}$. Moreover, if not all local $C R$ orbits of some connected representative of $(M, 0)$ are of codimension 2 in $M$, then $(H, 0)$ is unique.

Proof of Lemma 4.1. Let $S$ be the set of CR singularities of $M$. We note that $\mathcal{O}\left(\mathbb{C}^{N}\right)$-convex sets are precisely those that are polynomially convex. For a proof of this fact, see for example Rossi [17] (Lemma 2.4). Hence we can apply Theorem 1.1 to see that $H^{\circ}$ must be real analytic.

We find a point $p \in M_{C R}$ where the CR orbit is of codimension 1. Such a point exists by the hypothesis and by Lemma 3.2. We then apply Theorem 4.2 so that 
we get a real algebraic subvariety (i.e. defined by a real polynomial) $\mathcal{H} \subset \mathbb{C}^{N}$ that contains $M$. By the uniqueness of the germ and analytic continuation we know that $H \subset \mathcal{H}$.

We can now apply Theorem 2.2 to know that $H$ extends analytically past 0 and hence extends as a real algebraic Levi-flat hypersurface. We know that such hypersurfaces are locally given by an equation of the form $\operatorname{Im} w=0$ in some local coordinates $(z, w) \in \mathbb{C}^{N-1} \times \mathbb{C}$. Since $M$ is $\mathrm{CR}$ singular at 0 , we must have that $\frac{\partial}{\partial(\operatorname{Re} w)}$ is not tangent to $M$ at 0 , and hence the equations

$$
\operatorname{Re} w=\varphi(z, \bar{z}) \text { and } \operatorname{Im} w=0
$$

define $M$ near 0 .

Proof of Theorem 1.2. Let $M, H$ be as in the statement of Theorem 1.2. We first apply Theorem 1.1 to ensure that $H^{o}$ is real analytic. From the proof of Theorem 3.1 we know that all local CR orbits of $M_{C R}$ are of codimension 1 in $M$, and further that $H$ extends as a Levi-flat hypersurface past all the CR points of $M$. It remains to show that $H$ extends near the CR points. We need only apply Lemma 4.1 as we have now satisfied all the needed hypotheses.

\section{HyPERSURFACES WITH SINGULARITIES}

It is possible that there might exist a singular hypersurface $H$ with boundary $M$, even if $M$ is nonsingular. For example, we note that the Levi-flat subvariety $\operatorname{Im}\left(z^{2}+w^{2}\right)=0$ has an isolated singularity at 0 . If we also consider the inequality $|z|^{2}+|w|^{2} \leq 1$, then we have a hypersurface with a singularity at 0 and a nonsingular real analytic boundary. This example can be generalized to $\mathbb{C}^{N}$ for $N \geq 3$ in the obvious way.

Similarly, the existence result of Dolbeault, Tomassini and Zaitsev [10] does not guarantee a nonsingular hypersurface. An analysis of the proof of Theorem 3.1 allows us to formulate an alternative statement which allows singularities.

Theorem 5.1. Let $M \subset X$ be a compact real analytic submanifold of codimension 2. Suppose there exists a compact connected set $H$, and a closed set $E \subset H$, such that $H \backslash E$ is a $C^{\infty}$ Levi-flat hypersurface with boundary, and $\partial(H \backslash E)=M \backslash E$.

Let $S$ be the set of $C R$ singularities of $M$ and let $\widehat{S \cup E}$ be the $\mathcal{O}(X)$-convex hull of $S \cup E$. Then $H \backslash((\widehat{S \cup E}) \cup M)$ is real analytic.

In particular, if both $S$ and $E$ are finite sets. Then $H \backslash(E \cup M)$ is real analytic.

Proof. We notice that the only part of the proof of Theorem 3.1 that needs to be modified is the application of Lemma 3.5. Instead of $S$ we need to take the set $S \cup E$. We can then apply Lemma 3.5 to find a point $p \in B \cap(\widehat{S \cup E})$ and a peaking function $f$. We note that $p \in H \backslash(M \cup(\widehat{S \cup E}))$ and derive a contradiction in the same manner as in the proof of Theorem 3.1 .

Similarly, notice that we can modify Theorem 1.2. Again, we note that the $\mathcal{O}\left(\mathbb{C}^{N}\right)$-convex hull is the same as the polynomially convex hull, and we denote this convex hull of a set $K$ by $\hat{K}$. 
Theorem 5.2. Let $M \subset \mathbb{C}^{N}, N \geq 3$, be a compact real algebraic submanifold of codimension 2. Suppose there exists a compact connected set $H$, and a closed set $E \subset H$, such that $H \backslash E$ is a $C^{\infty}$ Levi-flat hypersurface with boundary, and $\partial(H \backslash E)=M \backslash E$.

Let $S$ be the set of $C R$ singularities of $M$. If $H \backslash(\widehat{S \cup E})$ is connected and $M \backslash E$ is nonempty, then there exists a real algebraic Levi-flat subvariety $\mathcal{H} \subset \mathbb{C}^{N}$ of codimension 1 such that $H \backslash(\widehat{S \cup E}) \subset \mathcal{H}$.

In particular, if $S$ and $E$ are finite sets, then the theorem applies and we conclude that $H \subset \mathcal{H}$. In this case the theorem says that $H$ is actually contained in a Levi flat subvariety even at points of $E$.

Proof. First we apply Theorem 5.1 to show that $H \backslash((\widehat{S \cup E}) \cup M)$ is real analytic. Since $M \backslash E$ is nonempty, then $M_{C R} \backslash E$ is nonempty and we find a point $p \in M_{C R}$ where we can apply Theorem 4.2 to find $\mathcal{H}$. That $H \backslash(\widehat{S \cup E}) \subset \mathcal{H}$ follows by analytic continuation.

\section{EXAMPLES}

To illustrate the ideas in the main theorems, we give the following examples. First in Example 6.1 we give the model example when the theorems are true. In Example 6.2 we show that uniqueness does not hold in two dimensions. Example 6.3 shows that near CR singular points, neither regularity nor uniqueness holds locally without extra hypotheses. Further examples of local behavior of Levi-flat hypersurfaces near a CR boundary can be found in [14.

Example 6.1. Suppose that $(z, w) \in \mathbb{C}^{n} \times \mathbb{C}$ are our coordinates and

$$
M:=\left\{(z, w) \mid \operatorname{Im} w=0,\|z\|^{2}+(\operatorname{Re} w)^{2}=1\right\},
$$

where $\|\cdot\|$ is the standard Euclidean norm on $\mathbb{C}^{n}$. $M$ has two CR singularities, at $(0, \pm 1)$. Then obviously $H$ is defined by

$$
H:=\left\{(z, w) \mid \operatorname{Im} w=0,\|z\|^{2}+(\operatorname{Re} w)^{2} \leq 1\right\} .
$$

It is clear that $H$ extends past its boundary as Theorem 1.2 implies.

Example 6.2. The failure of uniqueness on $\mathbb{C}^{2}$ is illustrated by the following example. Suppose that $(z, w) \in \mathbb{C}^{2}$ are our coordinates and

$$
M:=\{(z, w)|| z|=| w \mid=1\} .
$$

There are two obvious compact Levi-flat hypersurfaces with boundary $M$,

$$
\{(z, w)|| z|=1,| w \mid \leq 1\} \text { and }\{(z, w)|| z|\leq 1,| w \mid=1\} .
$$

There is also a singular Levi-flat hypersurface with boundary $M$,

$$
\{(z, w)|| z|=| w|,| z \mid \leq 1\} .
$$

Example 6.3. Let us see why local regularity need not necessarily hold near CR singular points if we do not insist on the hypersurface being compact. 
First let

$$
\varphi(x):= \begin{cases}e^{-1 / x} & x>0 \\ 0 & x \leq 0 .\end{cases}
$$

Let $(z, w) \in \mathbb{C}^{2} \times \mathbb{C}$ be our coordinates. Let $H$ be a Levi-flat hypersurface with boundary defined by

$$
\begin{aligned}
& \operatorname{Im} w=\varphi(-\operatorname{Re} w), \\
& \operatorname{Re} w \leq\left|z_{1}\right|^{2}+\left|z_{2}\right|^{2} .
\end{aligned}
$$

Note that the boundary of $H$, let us call it $M$, is defined by

$$
\operatorname{Re} w=\left|z_{1}\right|^{2}+\left|z_{2}\right|^{2} \text { and } \operatorname{Im} w=0 \text {. }
$$

Outside of the origin, $M$ is a CR submanifold, where the codimension in $M$ of the CR orbits must be 1 , as $M$ contains no complex analytic subvarieties. $M$ is in fact a real algebraic submanifold, while $H$ clearly is not real analytic near 0 .

By multiplying $\varphi$ by a parameter we see that there are infinitely many smooth Levi-flat hypersurfaces with boundary $M$. On the other hand, because the CR orbits are of codimension 1, Lemma 3.3 and analytic continuation show that there are only two connected real analytic Levi-flat hypersurfaces with boundary $M$. One is defined by $\operatorname{Im} w=0$ and $\operatorname{Re} w \leq\left|z_{1}\right|^{2}+\left|z_{2}\right|^{2}$, and the other by $\operatorname{Im} w=0$ and $\operatorname{Re} w \geq\left|z_{1}\right|^{2}+\left|z_{2}\right|^{2}$.

\section{REFERENCES}

[1] M. Salah Baouendi, Peter Ebenfelt, and Linda Preiss Rothschild, Real submanifolds in complex space and their mappings, Princeton Mathematical Series, vol. 47, Princeton University Press, Princeton, NJ, 1999. MR1668103

[2] Eric Bedford, Levi flat hypersurfaces in $\mathbf{C}^{2}$ with prescribed boundary: stability, Ann. Scuola Norm. Sup. Pisa Cl. Sci. (4) 9 (1982), no. 4, 529-570. MR0693779

[3] Eric Bedford and Bernard Gaveau, Envelopes of holomorphy of certain 2-spheres in $\mathbf{C}^{2}$, Amer. J. Math. 105 (1983), no. 4, 975-1009. MR0708370

[4] Edward Bierstone and Pierre D. Milman, Semianalytic and subanalytic sets, Inst. Hautes Études Sci. Publ. Math. 67 (1988), 5-42. MR0972342

[5] Edward Bierstone and Pierre D. Milman, Arc-analytic functions, Invent. Math. 101 (1990), no. 2, 411-424. MR.1062969

[6] Errett Bishop, Differentiable manifolds in complex Euclidean space, Duke Math. J. 32 (1965), 1-21. MR0200476

[7] Albert Boggess, CR manifolds and the tangential Cauchy-Riemann complex, Studies in Advanced Mathematics, CRC Press, Boca Raton, FL, 1991. MR.1211412

[8] John P. D'Angelo, Several complex variables and the geometry of real hypersurfaces, Studies in Advanced Mathematics, CRC Press, Boca Raton, FL, 1993. MR.1224231

[9] Klas Diederich and John E. Fornaess, Pseudoconvex domains with real-analytic boundary, Ann. of Math. (2) 107 (1978), no. 2, 371-384. MR0477153

[10] Pierre Dolbeault, Giuseppe Tomassini, and Dmitri Zaitsev, On boundaries of Levi-flat hypersurfaces in $\mathbb{C}^{n}$, C. R. Math. Acad. Sci. Paris 341 (2005), no. 6, 343-348. MR2169149

[11] Xiao Jun Huang and Steven G. Krantz, On a problem of Moser, Duke Math. J. 78 (1995), no. 1, 213-228. MR.1328757

[12] Hon Fei Lai, Characteristic classes of real manifolds immersed in complex manifolds, Trans. Amer. Math. Soc. 172 (1972), 1-33. MR0314066

[13] Jiří Lebl, Nowhere minimal CR submanifolds and Levi-flat hypersurfaces, J. Geom. Anal. 17 (2007), no. 2, 321-342. MR 2320166

[14] Extension of Levi-flat hypersurfaces past CR boundaries, Indiana Univ. Math. J. 57 (2008), no. 2, 699-716. MR2414332 
[15] B. Malgrange, Ideals of differentiable functions, Tata Institute of Fundamental Research Studies in Mathematics, No. 3, Tata Institute of Fundamental Research, Bombay, 1967. MR0212575

[16] Jürgen K. Moser and Sidney M. Webster, Normal forms for real surfaces in $\mathbf{C}^{2}$ near complex tangents and hyperbolic surface transformations, Acta Math. 150 (1983), no. 3-4, 255-296. MR0709143

[17] Hugo Rossi, Holomorphically convex sets in several complex variables, Ann. of Math. (2) 74 (1961), 470-493. MR0133479

Department of Mathematics, University of Illinois at Urbana-Champaign, Urbana, ILLINOIS 61801

E-mail address: jlebl@math.uiuc.edu

Current address: Department of Mathematics, University of California at San Diego, La Jolla, California 92093-0112

E-mail address: jlebl@math.ucsd.edu 